\title{
An Intramuscular Hemangioma at the Cervical Muscle: A Case Report
}

\author{
Kyoung-Min Jang, Seung-Won Park, Young-Baeg Kim \\ Department of Neurosurgery, Chung-Ang University College of Medicine, Seoul, Korea
}

Intramuscular hemangioma (IMH) primarily develops in the skeletal muscles of the limbs or trunk. The occurrence of IMH in the neck muscle is very rare. In the present report, we describe a case of IMH in the posterior neck muscle. A 58-year-old woman presented with a mass on the right posterior neck area, which had developed 2 years previously. The tumor was round in shape, had a size of $3.5 \times 3.0 \mathrm{~cm}$, and was fixed to the surrounding soft tissue. The patient complained of mild dull pain and tenderness at the mass without reddish discoloration. The mass showed a diurnal variation in size - the mass grew in size in the morning and became smaller in the afternoon. Preoperative study confirmed the vascular nature of the tumor. During peritumoral dissection, the mass shrank rapidly following profuse bleeding and arterial cauterization, and hence, it was difficult to distinguish the mass from the surrounding tissues. A total resection was possible with careful dissection of its fibrotic boundary. Based on the histological findings, a definitive diagnosis of cavernous type IMH was made. An initial suspicion of $\mathrm{IMH}$, according to the clinical findings, would be helpful for decision making of further evaluations and surgical plan.

Key Words: Hemangioma $\cdot$ Cavernous $\cdot$ Neck muscles

\section{INTRODUCTION}

Intramuscular hemangioma (IMH) is a rare type of benign tumor that accounts for $0.8 \%$ of all cases of hemangioma ${ }^{19)}$. It typically develops in skeletal muscles in the limbs or trunk, and sometimes occurs in the skeletal muscles in the head and neck area; the head and neck IMH's account for approximately $14-21 \%$ of all IMH cases ${ }^{6}$. The occurrence of IMH in the head and neck area is known to primarily involve the masseter and trapezius muscles ${ }^{3,9)}$. Moreover, most cases are often asymptomatic, as the tumor is usually painless, lacks skin lesions, and grows slowly ${ }^{14}$. In the present report, we describe the clinical characteristics, diagnostic procedures, and treatment of a case of IMH that developed in an unusual location, the semispinalis capitis muscle.

- Received: August 14, 2015 - Revised: September 4, 2015

- Accepted: September 4, 2015

Corresponding Author: Seung Won Park, MD, PhD

Department of Neurosurgery, Chung-Ang University Hospital, Chung-Ang

University College of Medicine, 102 Heukseok-ro, Dongjak-gu, Seoul

06973, Korea

Tel: +82-2-6299-3190, Fax: +82-2-6299-2069

E-mail: nspsw@cau.ac.kr

®This is an Open Access article distributed under the terms of the Creative

Commons Attribution Non-Commercial License (http://creativecommons.org/ licenses/by-nc/3.0/) which permits unrestricted non-commercial use, distribution, and reproduction in any medium, provided the original work is properly cited.

\section{CASE REPORT}

A 58-year-old woman from Mongolia presented with a palpable mass at the right posterior neck, which has been growing gradually over two years. She complained intermittent mild pain around the mass. Physical examination indicated that the mass had a diameter of approximately $5 \mathrm{~cm}$ and was soft and fixed to the surrounding tissues and accompanied by mild tenderness. Moreover, the mass showed a diurnal variation in size - it became larger in the morning and smaller in the afternoon. Simple neck radiograph didn't show any abnormal finding. Ultrasonography indicated the presence of an isoechoic multiloculated mass in the right semispinalis capitis muscle, whereas magnetic resonance imaging (MRI) showed a welldefinedlobulated and heterogeneous mass with strong contrast enhancement (Fig. 1). On angiography, a vascular staining was noted during the late arterial phase without any definitive feeding artery or draining vein.

Tumor removal was performed through a vertical posterior neck incision in the right posterior region of the neck. During the surgery, there was an episode of sudden profuse bleeding while dissecting the muscles surrounding the tumor. The bleeding was controlled easily with electrocautery, but the mass shrank in size. Consequently, the tumor boundary became unclear making the tumor dissection difficult. However, a total resection of the tumor was possible by careful dissection of the fibrotic plane around the tumor. The excised mass was 

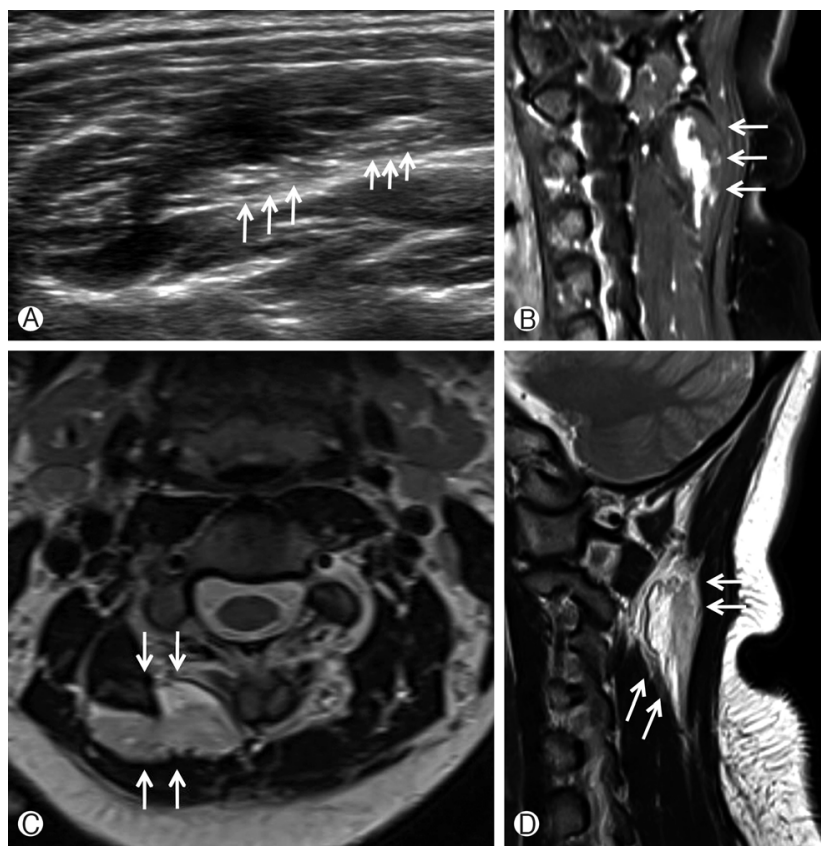

Fig. 1. (A) Ultrasonography shows a well-defined lobulated mass with isoechoic multiloculated mass in the right semispinalis capitis muscle (white arrows). (B) Sagittal T1-weighted contrast enhanced MRI shows a well-defined intramuscular mass with irregular centrifugal enhancement (white arrows). (C, D) Axial and sagittal T2weighted MRI show a well-defined lobulated mass with heterogeneous signal intensity (white arrows).

approximately $3.5 \times 3.0 \mathrm{~cm}$ and dark brown with small cystic components. The mass was diagnosed as a cavernous type IMH according to a histopathological examination (Fig. 2).

\section{DISCUSSION}

Hemangioma is a relatively rare form of a benign congenital tumor $^{6,15)}$. In 1843, Liston reported the first case of IMH, which had developed in the semimembranous muscle of the popliteal space ${ }^{13)}$. Since then, IMH's have been found to develop in the skeletal muscles of the limbs or trunk, with only about $14-21 \%$ occurring in the head and neck area, ${ }^{3,9,19)}$. In approximately $\geq 60 \%$ of cases of IMH in the head and neck area, the trapezius or masseter muscle is involved ${ }^{7}$; in particular, the occurrence of IMH in the posterior neck area is very rare, with only 2 such cases being reported to date, the sternocleidomastoid muscle and splenius capitis muscle ${ }^{6,14)}$. Hence, we believe that the IMH in the current case is the first report of IMH developed in the semispinalis capitis muscle.

The causes of IMH may be congenital, traumatic, or related to hormone imbalance, but the exact causes are not clear yet. The high incidence rate of this condition in newborns suggests

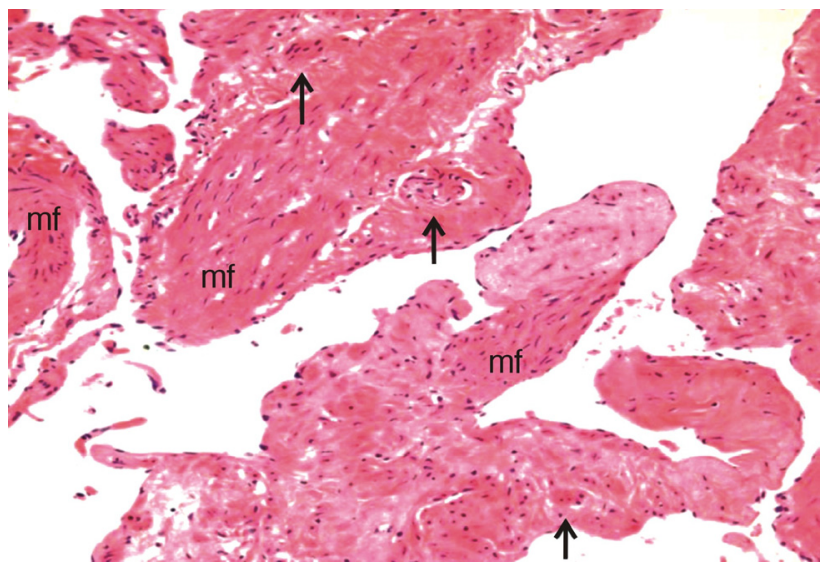

Fig. 2. Histological examination of cavernous type intramuscular hemangioma shows multiple vascular spaces lined by endothelial cells (black arrows) with striated muscle fibers $(\mathrm{mf})(\mathrm{H} \& \mathrm{E}$ stain, $\times 40)$.

its congenital nature, whereas the reported cases with IMH at the site of repeated soft tissue injuries may suggest a traumatic cause ${ }^{7,9,13)}$.

Due to its low prevalence and non-specific clinical symptoms, the preoperative diagnosis of IMH is known to be difficult $^{3,17)}$. The most common symptoms include pain and swelling, which is similar to the symptoms of other benign tumors ${ }^{20)}$. However, according to our case, a diurnal variation in size seems to be a helpful sign for IMH. To the best of our knowledge, the periodic size change has not been reported yet in association with IMH. In relation with other lesions, Avila et al. ${ }^{2)}$ described a diurnal variation in size in a case of lymphangioleiomyomatosis of the abdomen on CT. Moreover, in 12 cases of lymphangioleiomyomatosis, the size of the tumor was found to increase in the morning by approximately $140 \%$, compared to the size in the afternoon, as a result of the retention of lymph fluid during sleep. Although the IMH is not a lymphatic lesion, the mechanism of diurnal variation of the IMH size may be related with blood pooling in the mass during sleep. The tumor size increase in the morning may be related with blood pooling in the mass while lying down during sleep, and the size decrease in the afternoon as a result of daily activities. The decrease in blood flow in the tumor may be induced by the influence of gravity and contraction of surrounding muscles.

Among the diagnostic modalities, plain radiography mostly shows normal results, whereas accompanying calcification is noted in approximately $15 \%$ cases $^{11}$. Computed tomography indicates the presence of an enhanced and well-circumscribed lesion with external structures, although identifying the components within the tumor is known to be difficult $t^{4}$. MRI is a highly effective diagnostic modality for $\mathrm{IMH}^{10,14)}$. MRI findings of IMH include high-signal intensity findings on both 
T1- and T2-weighted images. In T2-weighted images, the tumor can be clearly distinguished from the normal surrounding muscle structures. Characteristic findings within the tumor include the presence of heterogeneous signal intensity as a result of an increased blood flow in the dilated tortuous vessels; this finding is quite important in the diagnosis process $^{10)}$

Allen conducted a study on 89 cases of hemangioma and reported that excessive blood loss can occur during surgery ${ }^{1)}$. To avoid such blood loss, preoperative angiography is necessory. If the exact structure and components of the tumor can be identified and a large feeder is present, measures such as embolization can be taken, in advance, to prevent unnecessary intraoperative blood loss and incomplete resection ${ }^{16}$.

IMH can be divided into 3 types, depending on the size of the vessels that constitute the IMH, and the clinical symptoms and recurrence rates can vary according to the types ${ }^{7,15}$. The capillary type consists mostly of small vessels; $30 \%$ of cases of capillary type IMH occur in the head and neck area. The characteristics of this type include the observation of pathologically distinctive endothelial nuclei and mitotic figures, as well as the presence of intraluminal projections on endothelial cells ${ }^{7,20)}$. The capillary type is the most common among the 3 types, has a relatively short disease duration, is often not accompanied by pain, and has a small size of $<10 \mathrm{~cm}$ in most cases. The local recurrence rate following surgical treatment of this type is $20 \%{ }^{20}$. The cavernous type is comprised of relatively larger vessels than the capillary type, and approximately $19 \%$ of cases of this type involve the head and neck area. This type is also associated with relatively longer disease duration and a larger size. Since pain is observed in most of these cases, it is easier to make an accurate diagnosis. The local recurrence rate after surgery in the cavernous type is $9 \%{ }^{20)}$. The mixed type involves a histological mix of capillary and cavernous types, with only $5 \%$ occurring in the head and neck area; the mixed type is associated with the highest recurrence rate among the 3 types with $28 \%{ }^{20}$. On histological analysis, the current case was the cavernous type of IMH, and its accompanying pain was helpful for diagnosis.

Although radiation therapy can be considered as a secondary therapeutic option for recurrent or invasive $\mathrm{IMH}$, its effect has not yet been clearly determined yet ${ }^{5,8,18)}$. The best treatment option to date is the complete surgical resection of the tumor ${ }^{11,12)}$. However, an excessive bleeding and sudden obscure of tumor boundary during dissection may be encountered during surgery, which can be overcome by preoperative evaluations for the tumor vascularity or exact intramuscular tumor location.

\section{CONCLUSION}

We report a case of an intramuscular hemangioma that developed in a rare location, the semispinalis capitis muscle. By performing preoperative evaluations with MRI and angiography, we could accurately diagnose the patient with IMH, and could perform a total tumor resection successfully. Moreover, the diurnal size variation of the tumor seems to be an important clinical sign helpful for early diagnosis.

\section{REFERENCES}

1. Allen P, Enzinger FM: Hemangioma of skeletal muscle. An analysis of 89 cases. Cancer 29:8-22, 1972

2. Avila NA, Bechtle J, Dwyer AJ, Ferrans VJ, Moss J: Lymphangioleiomyomatosis: CT of Diurnal Variation of Lymphangioleiomyomas. Radiology 221:415-421, 2001

3. Beecham JE, Behrens HW: Intramuscular hemangioma of the head and neck: report of a case involving the prevertebral muscles. Head Neck Surg 10:402-405, 1988

4. Buetow P, Kransdorf M, Moser Jr R, Jelinek J, Berrey BH: Radiologic appearance of intramuscular hemangioma with emphasis on MR imaging. AJR Am J Roentgenol 154:563-567, 1990

5. Chaimoff C, Lurie H: Hemangioma of the rectum: clinical appearance and treatment. Dis Colon Rectum 21:295-296, 1978

6. Chaudhary N, Jain A, Gudwani S, Kapoor R, Motwani G: Intramuscular haemangioma of head and neck region. J Laryngol Otol 112:1199-1201, 1998

7. Clemis J, Briggs D, Changus G: Intramuscular hemangioma in the head and neck. Can J Otolaryngol 4:339-346, 1975

8. Edgerton MT: The treatment of hemangiomas: with special reference to the role of steroid therapy. Ann Surg 183:517-532, 1976

9. Giudice M, Piazza C, Bolzoni A, Peretti G: Head and neck intramuscular haemangioma: report of two cases with unusual localization. Eur Arch Otorhinolaryngol 260:498-501, 2003

10. Hawnaur J, Whitehouse R, Jenkins J, Isherwood I: Musculoskeletal haemangiomas: comparison of MRI with CT. Skeletal Radiol 19:251-258, 1990

11. Hoehn JG, Farrow GM, Devine KD, Masson JK: Invasive hemangioma of the head and neck. Am J Surg 120:495-500, 1970

12. Jani P, Robb P, Hanby A, Gleeson M: Intramuscular hemangioma of the sternomastoid muscle. Head Neck 12:164-167, 1990

13. Jones KG: Cavernous hemangioma of skeletal muscle. J Bone Joint Surg 35:717-725, 1953

14. Makeieff M, Maurice N, Mondain M, Crampette L, Guerrier B: Intramuscular hemangioma of posterior neck muscles. Eur Arch Otorhinolaryngol 258:28-30, 2001

15. Mulliken JB, Glowacki J: Hemangiomas and vascular malformations in infants and children: a classification based on endothelial characteristics. Plast Reconstr Surg 69:412-420, 1982

16. Rossiter JL, Hendrix RA, Tom LW, Potsic WP: Intramuscular 
hemangioma of the head and neck. Otolaryngol Head Neck Surg 108:18-26, 1993

17. Scott J: Hemangiomas in skeletal muscle. Br J Surg 44:496-501, 1957

18. Stofman G, Reiter D, Feldman M: Invasive intramuscular hemangiomas of the head and neck. Ear Nose Throat J 68:612-616,
1989

19. Wild AT, Raab P, Krauspe R: Hemangioma of skeletal muscle. Arch Orthop Trauma Surg 120:139-143, 2000

20. Wolf GT, Daniel F, Krause CJ, Kaufman RS: Intramuscular hemangioma of the head and neck. Laryngoscope 95:210-213, 1985 